mitzutheilen, als cine eigene Lähmungsform, charakterisirt durch die Art ihres Auftretens - ganz allmäbliche Entwickelung in zweiten Lebensjahr - durcb ihre Verbindung mit Störungen der geistigen Entwickelung, insbesondere der Sprache, durch die Art der motorischen Störungen - Parese in allen Extremitäten bei erhaltener electromusculärer Contracticität, - durch die grosse Funktionsstörung und den tabesähnlichen Gang, und endlich durch die Abwesenheit aller Sensibilitätsstörungen.

Ich habe, seitdem ich Obiges niedergeschrieben, in der pädiatrischen Litteratur wohl noch Manches über Gehirnerweichung bei Kindern gefunden, allein Nichts was unseren Fällen auch nur entfernt gleich käme. Mag die Zeit, in der dicse Worte geschrieben worden, entschuldigen, dass ich jene Fälle nicht ausführlich bespreche. Hamburg, 16. Juli 1870.

\title{
VII.
}

\section{Multiple Fibrosarkome der Nerven und Perichondritis laryingea.}

\author{
Von Johannes Seitz, \\ Assistenten der medicitischen klinik in Zürich.
}

Der folgende Fall, welcher auf der medicinischen Klinik von Herrn Professor Biermer zúr Beobachtung kam, mag einen Beitrag liefern zur Entscheidung der Frage, in wie weit die in der Continuität der Nerven multipel vorkommenden und als Neurome bezeichneten Geschwülste den wahren Neuromen oder den Fibromen müssen zugerechnet werden.

Krankengeschichte.

J. H. Keller, 49 Jahre alt, Landarbeiter von Mönchaltdorf, wurde den 8. Juni 1869 auf die med. Klinik aufgenommen. Er starb schon am folgenden Tage Nachts 11 Uhr $15 \mathrm{Min}$.

Die frïheren Gesundheitsverhaltnisse des Pat. konnten nur mangelhaft theils von ihm selbst, theils von seinem Bruder und zwar zum grössten Theile erst nach des Kranken Tode erboben werden.

Körperlich war Pat, häufig auftretende Furunkel ausgenommen, stets vollkommen gesund. Geistig zeigte er schon in früher Jugend Abnormitäten. Stets 
war er ein Sonderling; vom 20. bis 30 . Jahre häufig aufgeregtes Wesep, grosser Jähzorn; manchmal steigerte sich die Aufregung bis zu leichten Tobsuchtsanfällen. Nach diesen Aufregungsstadium wurde Pat. immer einfaltiger. Im Ganzen zeigte sich ein ungemeiner Wechsel seiner Stimmungen. Bald war er gut gelaunt und machte dann curiose Witze, sonderbare Reimschneidereien; bald entwickelte er die wunderlichsten Ideen; für kurze Zeit konnte er vernünftig wie ordentliche Leute sich aufführen. Mehrmals machte er sich verschiedener Diebereien an einem Bruder schuldig, bei dem er die letzten 16 Jahre sich aufhielt. Er konnte nur zur Landarbeit verwendet werden. War er bei guter Laune, so arbeitete er auf gute Worte ordentlich, in störrischen Zeiten musste er durch Drohungen und Prügel daza gezwungen werden. Oft sammelte er Tage lang Glasscherben und Holzstückchen.

Seit sieben Jahren (1862) litt Pat. an Beengung, deren Grad bedeutend wechselte. Anfangs trat sie nur bei Anstrengungen auf, nachber auch in der Rube; im Laufe der Jahre steigerte sie sich beständig, doch früher nie zu einer beunruhigenden Höbe. Bis qor zwei Jaluren war Pat. fett und stark gewesen, „180 Pfund schwer, ein Bursch wie eine Eiche," von da an magerte er ab zu einer langen bageren Gestalt; besonders seit Sommer 1868 hat diese Abmagerung zugenommen, für weiche die Ungebung keinen Grund sich denken konnte, da das Allgemeinbefinden nicht gestört war.

Im Herbst 1868 und wieder im Frühling 1869 litt Pat. 4-6 Wochen lang an Heiserkeit, Athemnotb, „einem Gefühle, als ob ein Stäck Fleisch im Halse vor den Athem käme." Husten war vorhanden, doch nie war er besonders heftig. Der ziemlich reichliche dickschleimige Auswurf wurde während dieser Anfälle von Heiserkeit leicht entleert. Nie waren dem Auswurf Knorpelstückchen, Blat, auffallende Ejtermassen beigemengt. Pat. batte gar nie Kiagen über irgend etwas ausser über Beengung während der 4-6wöchentlichen Heiserkeil; gar nie Schmerzen im Kehlkopfe; nie Schluckbescbwerden.

In den letzten 4 Wochen vor dem Eintritt in's Spital, seit Anfang Mai 1869, wieder Heiserkeit, Beengung, keuchende, rasselnde Respiration; keine weiteren Beschwerden. Seit Freitag 4. Juni 1869 so bedeutende Zunahme der Athembeschwerden, dass Put. sowahl wie seine Umgebung den Erstickungstod als Ausgang befürchteten. Deshalb Eintritt in's Spital 8. Juni 1869.

Specifische Affectionen waren nie vorhanden gewesen.

In Bezug auf die zahlreichen Geschwülste gab Pat. an, dass er sie seit seiner Jugend besitze. Die grosse Geschwulst am linken Knie fiel dem Bruder erst vor 6 Jahren zufälligerweise auf. Wie lange vorher sie schon bestanden, ob die Grösse sich vorher oder nachher veràndert habe, wusste er nicht anzugeben. Pat. zeigte nie irgend welche Bewegungsstörungen und klagte gar nie über Anăsthesien, nie äber irgend welche Schmerzen; bei der Ontersuchung jedoch war Drack auf die verschiedenen Tumoren sehr schmerzhaft.

Nach den Angaben des Bruders ist Niemand aus der ganzen Verwandtschaft mit ahnlichen Geschwïlsten behaftet und Pat. das einzige Glied der Familie, welches je psychische Störungen zeigte.

Pat. bot bei seinem Eintritte 8. Juni 1869 die Zeichen hochgradiger Athemnoth. Fast heständig sass er aufrecbt im Bette, oder ging herum, respirirte mit 
grasser Anstrengung; In- und Exspiration waren sehr geräuschvoll. Am 9. Juni 1869 nabm die Abemnoth bedeutend za. Am Besten fassen wir die Erscheinungen, welche Pat. bot, zusammen durch Wiedergabe der klinischen Besprechungen durch Herrn Prof. Biermer. Langes, hageres Individuum; kräftiger Bau; schlechte Ernährung; leichtes Oedem der Fände; Venenschwellung am Halse; Livor an Lippen, Wangen, Ohren. Am auffallendsten ist die Athmungsweise des Pat. Alle Athemmuskein werden in höchstem Grade angestrengt. Hals-, Zwisehenrippen-, Bauchmuskeln und Zwerchfell arbeiten ungemein beftig. Die Inspiration ist selar lang gedehnt, die Exspiration auch verlängert, doch in geringerem Grade. Die untere Apertur des Thorax, Epigastrium, Supraclaviculargruben ziehen sich ein, der Kehlkopf steigt tief herunter bei jeder Inspiration. Es muss ein sebr grosses Athmungshinderniss bestehen entweder im Larywx oder in der Trachea, um so hochgradige Athembewegungen, so bedeutende Luftverdünnung in Thorax zu bewirken, dass ein 49jäbriger, schon recht seniler, starrer Brustkorb noch in solchem Grade durch den äusseren Luftdruek comprimirt wird. - Beim Husten bläht sich der Hals auf und die Dyspnoe steigert sich. Die Quesfurche vom Transversus abdominis ist bei der Exspiration sebr ausgesprochen. Im ganzen Saale sind bei der Respiration des Pat. Geräusche zu hören; ein eigenthümliches pfeifend schnurrendes langgezogenes Geräusch bei der laspiration, bei der Exspiration höheres, kürzeres, ähnliches Geräusch. Die Untersuchung des Halses ergibt: die Gland. thyreoidea links geschwellt; da ragt ein derber Zipfel des Kropfes, dessen unteres Ende sich unter dem Steraum verliert, bis an die Carti, thyreoidea hinauf; auf der rechten Seite der Trachea ist keine ScbwelJung der Scbilddrüse vorbanden. Der Kehlsopf ist etwas nach rechts gedrängt. Eine Schmerzhaftigkeit desselben bei Berührung besteht nicht. Die Sprache ist rauh, heiser, schwach, wegen der Dyspnoe absatzweise.

Die Percussion des Thorax ergibt airgends Dämpfung, sonoren Lungenschall in normaler Ausdehnung. Herz-, Leber-, Milz-Dämpfung sind normal; im Abdomen nirgends pathologische Dảmpinng. Temperatur, Stubi und Urin normal. Ziemlich viel dünnschleimiger, schaumiger Auswurf ohne besondere Beimengungen. Die Percussionsresultate sind auch hinten am Thorax normal. Bei der Inspection zeigt sich die Wirbelsăule etwas nach rechts verbogen; gute Wölbung des Thorax; starke Athemanstrengungen, starke inspiratorische Einziehung der unteren Rippen. Nirgends auf der Lmbge is vesiculäres Athmen zu bören; allenthalben ein ungemein lautes tiefes brummendes Gerăusch ăholich dem "Muben“ der Kühe bei der Inspiration, ejn hohes. Pfejfen bei der. Exspiration. Durch das Stethoskop sind diese Geräusche weniger hörbar als beim Auflegen des blossen Ohres. Die Herztöne sind für gewöhnlich von den lauten Respirationsgeräuschen ganz verdeckt; wenn der Pat. angebalten wird, einen Augenblick nicht za athmen, sind sie als rein zu erkennen.

Die Laryngoskopie wird vorgenommen, ergibt aber kein Resultat; denn der bestăndig zum Keblkopf herausgehustete Schleim, die beständige Unrube des Pat., seine Unfäbigkeit den Athem anzubalten, verhindern einen Einblick.

Herr Prof. Biermer sprach sich für Tracheostenose durch Struma aus, besonders weil die Palpation am Halse sehr wahrscheinlich machte, dass die Gland. thyreoidea noch unter das Sternum reichte und die Erfahrung genugsam lebrt, wie häufig diese substernalen Krö̈pfe Verengung der Luftwege bewirken. Die Möglichkeit 


\section{7}

eines Glottiödems musste offen erbalten bleiben, obschon kein denkbarer Grund für ein solches vorlag, und vom Munde aus die Vorderwand der Epiglottis als normal sichtbar war. Die laryngoskopische Untersuchung hatte eben die nothwendige sichere Entscheidung nicht gebracht.

Am Körper des Pat. fanden sich ansserdem zahlreiche Knoten vor, ein faustgrosser Tumor in der linken Kniekeble, zahlreiche kleinere erbsen- bis wallnussgrosse an Rumpf, Armen und Beinen. Ibre dem Verlaufe der Nerven entsprecbende Lage, die Schmerzhaftigkeit einzelner bei Druck hatten den Verdacht von Neuromen erweckt. Doch konnte wegen der Tracheostenose der Verdacht auf einen bösartigen Mediastinaltumor mit Metastasen unter die Haut nicht ausgeschlossen werden. Der consultirte chirurgische College verschob darum die Tracheotomie bis diese Frage sicher beantwortet wäre. Das schnelle Ende des Pat. kam aber dazwischen. Mittwoch den 11. Juni Nachts $11 \mathrm{Uhr}$ war Pat. noch in verhältnissmässig ordentlichem Zustande; Nachts $11 \frac{1}{4}$ Ubr starb er nach einer Urinentleerung plötzlich.

Die Behandlung hatte bestanden in einem Aderlass von Unc. viij, kalten Compressen über den Hals, Inhalationen von Wasserdämpfen, Morphium - Alles war olne Effect gewesen.

Section 10. Juni $186911 \frac{1}{2}$ Uhr Morgens.

Cadaver in voller Todtenstarre. Die feinen Gefässe der Pia sind mässig injicirt; das Gehirn blass und feucht.

An Hals e zeigt sich unter dem M. sternocleidomastoideus beider Seiten eine stark vergrösserte Gl. thyreoidea. Der M. sternohyoidens beiderseits abgeplattet und durch die vergrösserte Thyreoidea auf die Seite geschoben. Thyreoidea allseitig vergrössert; die mittlere Partie, welche beide Lappen verbindet, stark prominent, weit heruntergehend. Die Tbyreoidea umfasst die Trachea so, dass man von ihr vorläufig nichts sehen kann. Der mittlere Lappen geht bis $1 \frac{1}{2}$ Linien unter die untere Grenze des lingknorpels. Nachdem der KehIkopf mit der Trachea und Thyreoidea herauspräparirt ist, sieht man die seitliche Compression der Trachea durch die Struma bis 1 Zoll unterhalb des Kehlkopfes hinabreichend. In den Oesophaguseingang ragen die schlotternden ödematōsen Wälste der Schleimhaut des Keblkopfeinganges. Die Schleimhaut über den Ligg. aryepiglottica, cart. arytaenoides und Santorini ist ziemlicb stark ödematös infiltrirt, der Kelılkopfeingang dadurch mässig beengt. Die Stimmbänder sind schlaff, nicht ödematös. Die Schleimhaut der Taschenbănder und des Kebldeckels ist succulent, ibre Follikel offenbar etwas grösser als normal. Unterhalb des ödemạtösen Wulstes des Oesophaguseinganges, an der hinteren. Fronte der Jinken Kellkopfshälfte ist die Oesophagusschleimhaut durchbrochen durch ein Geschwür vom Umfange eines Kirschkernes. Der nekrotische Ringknorpel schaut durch die Geschwürsöffnung heraus. Eiter entleert sich aus dem Geschwüre nicht. Mit der Sonde kommt man nicht tief hinter den entblössten Knorpel; auch kann die Sonde nirgends in's Keblkopfsinnere dringen. Das Struma ist parenchymatös, colloid.

In der recbten Lunge subpleurale Hämorrhagien. Aus dem Bronchus entleert sich dünnes blutiges schaumiges Secret. Bronchialschleimbaut stark injicirt. Lunge überall lufthaltig, mittlerer und unterer Lappen ödematös, ziemlich byperämisch. 
Linke Lunge zeigt mit Ausnahme der subpleuralen Hämorrhagien das Gleiche. Beiderseitig körnige Verdickung der Pleura.

An einer Aortalklappe einzelae kleine Verdickungen; im Herzen sonst nicbis Besonderes.

Milz vergrössert in die Dicke und Breite. Parenchym ziemlich fest, dunkelroth, Malpighi'sche Körper zahlreich und vergrössert.

Leber im rechten Lappen etwas blutreich; sonst keine nennenswerthen Veränderungen.

Nieren gross, ziemlich blutreich. Zahlreiche Geschwülste an zahlreichen Nerven, von der Grösse eines kleinen Kirschkerns bis zu der eines grossen Apfels.

Die Section hat also die klinische Diagnose Tracheostenose durch Struma bestätigt. Daneben bestand Gehirnoedem, als dessen Ursache trotz der Schmerzlosigkeit des Kehlkopfes eine Perichondritis laryngea sich ergab. Die ulcerirte Knorpelparthie hätte man laryngoskopisch wohl nicht erkannt, aber das Oedem des Kehlkopfeinganges hätte an Knorpelentzündung erinnern müssen, da häufig Oedeme der Kehlkopfschleimhaut ihren Grund in Entzündungsvorgängen an den unterliegenden Knorpeln haben. Die Perichondritis unseres Pat. war eine chronische; in Zusammenhang mit ihr entwickelte sich acut ein Oedem des Kehlkopfeinganges; dieses vermehrte die Dyspnoe von der Tracheostenose her colossal und bewirkte schliesslich den Tod.

Die Anordnung der Geschwülste war folgende:

Einzelne Anschwellungen fanden sich an folgenden 9. Nerven: an einem Jinkseitigen Lendennerven, am rechten und linken N. ulnaris, am rechten und linken N. cutaneus femoris externus, an einem Hautnerven innen am linken Oberarm, vorn am rechten 0 berschenkel und am linken und rechten Unterschenkel. Zahlreiche Geschwülste trugen die beiden N. ischiadici, Zunächst bei sorgfältigem Betrachten und Befühlen derselben entdeckte man 6 ganz kleine Knötchen. Ausser diesen fanden sich 7 grössere Tumoren. Von diesen sassen am rechten $N$. ischiadicus drei, einer an der Theilungsstelle in tibialis und peronaeus, einer an einem Seitenaste des $\mathrm{N}$. tibialis und einer am N. peronaeus. Der linke $\mathrm{N}$. ischiadicus trug vier Knoten, zwei Tumoren im Verlauf des Tibialisstammes, einer am N. suralis magnus, einer am N. peronaeus gerade in der linken Kniekehle.

Frei waren Gehirn und dessen Nerven, Rückenmark, Sympathicus, Plexus cervicobrachialis und lumbosacralis.

Es fanden sich somit 22 Anschwellungen an den verschiedenen Nerven des Körpers.

Der grösste Knoten ist der am linken $N$. peronaeus, welcher im Leben in der linken Kniekehle eine beträchtliche Geschwulst darstellte. $\mathrm{Er}$ ist von der Grösse einer Mannsfaust. Der zweitgrösste sass am rechten N. cutaneus femoris externus in der Grösse einer Baumnuss. Die meisten übrigen Knoten sind haselnussgross. 
Die 6 kleinsten Knötchen an beiden $N$. ischiadicis haben eine Länge von $0,5 \mathrm{Cm}$. und eine Dicke von $0,25 \mathrm{Cm}$.

Die Form der verschiedenen Tumoren ist kuglig, spindel - und eiförmig. Die kleinsten erscheinen als blosse spindelförmige Verdickung eines kleineren Nervenbündels in einem grösseren Nervenstamme, die man zuweilen besser beim Betasten als bei der Inspection findet.

Die Geschwülste haben von aussen betracbtet eine weisse Farbe ähnlich derjenigen der Nervenstämme. Sie sind mit diesen von einer bindegewebigen Scheide überzogen, die zuweilen zu einer derben Kapsel verdickt ist und als solche sich ziemlich leicht ablösen lässt.

Das Verhalten der einzelnen Nervenbündel zu den Geschwälsten des hetreffenden Nervenstammes ist folgendes. Es tritt bald an einem Ende eines Knotens „der Nerv" ein, am anderen Ende aus. Der Nerv scheint einfach durch die Geschwulst unterbrochen. Bei anderen Geschwälsten laufen die aus einander gedrängten und leicht abgeplatteten Nervenbündel über die Geschwulst hinweg, um sich unter derselben wieder zu einem Nervenstamme von gewöbnlichern Aussehen zu vereinigen. Andere Geschwülste sitzen mehr seitlich am Nerven. So treten an der grössten Geschwulst ebenfalls nur einzelne Nerventündel in den Tumor; der grösste Theil läıft flach ausgebreitet über den Tumor hin und verlässt die Geschwulst atrophisch, um sich in die zum grossen Theil untergegangenen Streckmuskeln des Fusses zu verbreiten.

Die Consistenz aller Geschwülste ist eine feste; sie fühlen sich derb elastisch an.

Auf dem Durchschnitt der Tumoren lassen sich virgends von blossem Auge durchgehends Nervenbïndel finden; wo solche in die Geschwulst eintreten, scheinen sie sich sogleich in's übrige Gewele zu verlieren. Die Farbe des Durchschnitts ist weisslich, ähnlich derjenigen der Oberläche. Einzelne Partien sind melir gelblich, derbere wechseln mit weicheren, markigen Partien ab. In einzelnen Tumoren finden sich kleine mit gelblicher schleimiger Masse gefüllte Höhlen.

Was zunäclst die kleinsten $\mathrm{Kn}$ ötchen von $0,5 \mathrm{Cm}$. Länge und $0,25 \mathrm{~cm}$. Dicke betrifft, so bestanden sie ganz wie die grossen Geschwülste aus einzelnen feinen Nervenbündeln, die über ein mittleres, spindelförmig verdicktes hinwegliefen. Die unverdickten und das verdickte Nervenbündel waren durch schr reichliches lockeres Bindegewebe und eine ziemlich dicke und derbe bindegewebige Scheide zu dem scheinbar einheitlichen Geschwülstchen vereinigt.

Die mikroskopische Untersuchung der Umhüllung zeigte nichts als gewöhnliches, lockeres, zellenarmes Bindegewebe. Die unverdickten Nerverbündelchen boten mässige Vermehrung des Neurilems, keine Spur von Vermehrung der Nervenfasern, vielmehr geringe Verfettung einzelner derselben. Das centrale Nervenbündelchen mit der Anschwellung ergab folgende Verhältnisse. Im Knötchen selbst wies das Mikroskop spärliche und zum Theil verfettete Nervenfasern in einem reichlichen fibrillären Bindegewebe 
nach. Im Querschnilt erschienen die einzelnen Nervenbündelchen weit auseinander gedrängt durch dicke Balken von Bindegewebe. Das ab- und zugehende Nervenstämmchen, nicht weiter zerlegbar, konnte direct unters Mikroskop gebracht werden; sie zeigten deutliche Vermehrung ihres Neurilems und Verfettung einzelner Nervenfasern. Aber nirgends eine Spur von Zunahme des Nervengewebes, auch nicht etwa kernreiche Fibrillen, die man als marklose Nervenfasern hätte deuten können. In den grösseren Geschwülsten ist es mir nirgends gelungen, trotz eifrigen Suchens, in Längsschnitten Nervengewebe aufzufinden. Auch die Proben mit Chlorgold ergaben Herrn Professor Eberth keine Nervenreaction. In Querschnitten waren bald Nervendurchschnitte zu erkennen, bald nicht. Es finden sich viele Partien, welche ganz und gar aus jungem Bindegewebe bestehen. In einer mässig reichlichen feinkörnigen Zwischensubstanz sind massenhafte junge rundliche Zellen eingebettet. An anderen Stellen findet sich spärliches mit Rundzellen durchsetztes leicht fibrilläres Bindegewebe. Zuweilen ist das Bindegewebe derber oder lockig und feinfibrillär:

Das junge Rundzellengewebe bịtet oft ausgezeichnet schöne Uebergänge zu netzförmigem Bindegewebe, indem die runden Zellen sich verlängern, ei- oder spindelförmig werden, an ihren Enden lange und verzweigte Ausläufer treiben, welche mit denen benachbarter Zellen zu einem zierlichen Netzwerk sich verbinden, in dessen Maschen stellenweise ziemlich grosse runde Zellen liegen.

Es ist aber an den meisten Geschwülsten der grösste Theil ein Rundzellengewebe, das einen wesentlich anderen Charakter besitzt als das oben erwähnte junge Bindegewebe mit den runden Zellen. Die Zellen sind grösser und zahlreicher, ihre Kerne bedeutender; zwischen jenen liegt eine ganz lockere, sehr spärliche Zwischensubstanz, die nur lose die einzelnen Zellen verbindet. . Diese Partien gleichen offenbar mehr dem Gewebe des Rundzellensarkoms als dem der Granulation. Daneben finden sich grosse Strecken von dem Baue des ächten Spindelzellensarkoms. Schmale Zellen liegen in reicher Zahl ohne erkennbares Zwischengewebe dicht aneinander und bilden obne alle Regelmässigkeit sich kreuzende Stränge. Mitunter finden sich neben den einzelnen spindelförmigen Elementen auch kleine Heerde runder Sarkomzellen.

Immerhin prävaliren in den grossen Geschwulsten die sarco- 
matösen Partien; nur einzelne Stellen können bestimmt als bindegewebig oder fibrosarcomatös bezeichnet werden.

Entschieden nur durch übermässige Vermehrung des Bindegewebes entstanden sind die kleinsten Geschwälste an beiden $\mathrm{N}$. ischiadicis, von $0,5 \mathrm{Cm}$. Länge und $0,25 \mathrm{~cm}$. Dicke, welche wohl den Anfang des Prozesses veransebaulichen. -

Man hat durchaus kein Recht irgendwo in den Nervengeschwülsten unseres Falles Vermehrung des eigentlichen Nervengewebes anzunehmen. Die Geschwülste sind Fibrome, Fibrosarkome und Sarkome.

Als Rückbildungsvorgänge zeigten einige Geschwülste Cystenbildung. Die gallertige Fullungsmasse derselben ist Schleimgewebe. In einzelnen derartigen Stellen haben kleine Hämorrhagien stattgefunden. Gelbe Durchtränkung der Gewebeelemente und Hämatoidinkrystalle weisen darauf hin.

In Betreff des Einflusses der Geschwülste auf die Nerven, an welchen sie sitzen, zeigt sich, dass eine Strecke weit aufwärts und abwärts am Nerven eine mässige Vermehrung der bindegewebigen Theile und eine theilweise Verfettung der Nervenfasern vorhanden ist. Einzig die faustgrosse Geschwulst am linken N. peronaeus, die in der Kniekehle sass, bewirkte theilweise den Untergang der Nervenfasern in den abgehenden Nerven. Diese stellen derbe Stränge vor, in welchen oben nur noch spärliche verfettete, weiter unten sehr schmale und markarme Nervenfasern nachweisbar sind. Die von den betrefienden Nerven versorgten Streckmuskeln des linken Fusses waren theils verfettet, theils ganz geschwunden und durch Bindegewebe ersetzt.

Mit mehreren der bekannten Fâlle stimmt der unsrige nicht allein im Bau, sondern auch in gewissen, während des Lebens beobachteten Störungen überein.

Wie bekannt waren viele Träger multipler Neurome geistig gestörte Individuen, Irre, Cretinen, Idioten. Unser Kranker war jedenfalls psychisch abnorm, wenn man auch nicht das Recht hat, ihn blos auf die Anamnese hin für einen Irren oder Idioten zu halten. In den 36 Stunden, die er hier beobachtet wurde, zeigte er sich allerdings nur als ein beschränkter Mensch.

ob die Neuromatosis familiär war, wie in vielen Fällen, ist in unserem Falle ungewiss. Die Angaben des Bruders lauten zwar. 
negativ; aber es kann doch seinem Urtheile nicht unbedingt Glauben geschenkt werden.

Es sind schon bei einer Reihe Syphilitischer Neurome entdeckt worden, ob in Zusammenhang mit der Lues, bleibe dahingestellt. Weder Anamnese noch Untersuchung gaben Anhalte, specifische Affectionen bei unserm Patienten anzunebmen.

Ausser Scbmerzhaftigkeit bei Druck, die wir constatiren konnten, verursachten die Geschwälste keine weiteren Symptome. Es hat dies nichts Auffallendes. Schon mehrere multiple Neurome sind erst an den Leichen entdeckt worden, indem sie nie die geringsten auffalligen Symptome machten. Bei andern konnten wohl die an Nerven sitzenden Knoten schon im Leben erkannt werden, aber weder spontaner Sehmerz, noch Lähmung von sensiblen oder motorischen Nerven war vorhanden. Das gewöhnlichste Symptom der Geschwülste ist Schmerzhaftigkeit bei Druck, wie sie unser Fall bot. Auch sehr gewöhnlich sind die spontanen Schmerzen, schon seltener Lähmung der sensiblen und motorischen Bahnen, Atrophie der versorgten Muskeln. In einzelnen Fällen sollen Erbrechen, Respirationsstörungen, Herzklopfen die Folge von Neuromen gewesen sein.

Die Anzahl der Geschwülste in unserem Falle ist keine bedeutende, sind ja schon tausende, über die verschiedensten Nerven zerstreut, gezählt worden. Genersich hat bei seinem in diesem Archiv (Bd. XLIX. H. 1. S. 15) veröffentlichten Falle die einzelnen Neurome nicht gezählt; die Zahl muss aber eine ungemein grosse gewesen sein, denn bis in die feinsten Verzweigungen sassen fast an allen Nerven zahlreiche Geschwülstchen.

Ueber die Art des Gewebes, welches die multiplen Neurome bildet, bestehen mannichfache Angaben. Bald sollen durch blosse hochgradige Vermehrung unzweifelhafter markhaltiger Nervenfasern, seltener von Ganglienzelleñ Geschwülste entstehen, bald wieder durch Wucherung von marklosen Nervenfasern und endlich durch Bindegewebe oder durch Sarkome oder Krebsgewebe. Die regressiven Veränderungen sind die Mucimetamorphose, Verkäsung, Verkalkung, Cystenbildung, oft mit Blutung in die Höhle.

Betonen möchte ich noch die Verschiedenheit im Baue der kleinsten und der grösseren Nervengeschwillste, die auch in dem von Genersich mitgetheilten Falle in ganz analoger Weise sich fand. Die kleinsten Tumoren bestehen aus fibrillärem Bindegewebe 
mit nicht vermehrten Nervenfasern, die grossen aus Bindegewebe mit Vebergang zum Sarkomgewebe - ebenfalls ohne Zunahme der Nerven.

Bemerkenswerth sind die Spindelzellen mil sehr langen Ausläufern. Diese Ausläufer für sich betrachtet, hätten leicht für junge Nervenfasern gebalten werden können und deshalb ein Theil der Neurome für wahre. Die genaue Untersuchung liess aber das Verhalten jener Fäden zu den Zellen mit Sicherheit erkennen.

Herrn Professor Eberth danke ich für die freundliche Unterstützung bei meinen Untersuchungen.

\section{VIII. \\ Zur Lelire von der Wärmeregulirung.}

Von Dr. C. Liebermeister, Professor in Basel.

Im XLV. Bande dieses Archivs (S. 351-413) findet sich eine Arbeit von Senator, deren einer Theil den $\mathrm{Zweck}$ hat, die von mir vertretene Ansicht von der Existenz einer Regulirung der Wärmeproduction nach dem Wärmeverlust za widerlegen. Ich habe es damals für tiberflüssig gehalten, in eine unfruchtbare Polemik einzutreten, da ich überzeugt war, jeder Sachverständige werde sofort erkennen, dass die mitgetheilten Versuchsresultate mit meinen Anschauungen in vollster Uebereinstimmung sich befinden, aber freilich für die Frage, um welche die angehängten rationalistischen Deductionen sich drehen, vollkommen irrelevant sind. Auch setzte ich voraus, dass der Verfasser selbst bei etwas eingebenderem Studium der betreffenden Verhältnisse das Unberechtigte und Trügerische seiner Ausführungen erkennen werde.

Letztere Erwartung ist, wie einzelne gelegentiche Auslassungen und namentlich ein längerer im L. Bande dieses Archivs (S. 354 bis 375) erschienener Artikel desselben Verfassers zeigen, bisher sicht in Erfüllung gegangen. Es werden ähnliche Versuche und ähnliche Schlussfolgerungen wiederholt; dann aber wird auch in der That der Kern der Frage beruhrt und durch Rechnung die Unrich- 